\title{
The CME-productivity associated with flares from two active regions
}

\author{
S. Akiyama ${ }^{\mathrm{a}, \mathrm{b}, *}$, S. Yashiro ${ }^{\mathrm{a}, \mathrm{b}}$, and N. Gopalswamy ${ }^{\mathrm{b}}$ \\ ${ }^{a}$ Department of Physics, the Catholic University of America, 200 Hannan Hall, \\ Washington, D.C. 20064, USA \\ ${ }^{\mathrm{b}}$ NASA Goddard Space Flight Center, Code 695.0, Greenbelt, MD 20771 USA
}

\begin{abstract}
We report on two flare-productive adjacent active regions (ARs), with different levels of coronal mass ejection (CME) association. AR 10039 and AR 10044 produced strong X-ray flares during their disk passages. We examined the CME association rate of X-ray flares and found it to be different between the two ARs. AR 10039 was CME-rich with 72\% association with flares, while AR 10044 was CME-poor with an association rate of only 14\%. CMEs from the CME-rich AR were faster and wider than the ones from the CME-poor AR. The flare activity of AR 10044 was temporally concentrated over a short interval and spatially localized over a compact area between the major sun spots. We suggest that different pre-eruption evolution and magnetic configuration in the two regions might have contributed to the difference between the two ARs.
\end{abstract}

Key words: Sun: flare, Sun: coronal mass ejection, Sun: activity

* Corresponding author.

Email addresses: akiyama@ssedmail.gsf .nasa.gov (S. Akiyama), 


\section{Introduction}

Previous investigations of the relationship between flares and coronal mass ejections (CMEs) have shown that flares with CMEs are intense and longlived. According to Yashiro et al. (2005), the CME association rate increases with X-ray flare size from only $20 \%$ for C-class to $100 \%$ for X3-class flares. Approximately half of M-class flares have associated CMEs, so the flare class by itself is not sufficient to determine whether a flare has a CME or not. There must be other discriminators between flares with and without CMEs. Canfield et al. (1999) found ARs with obvious sigmoidal (S-shaped) structure are more likely to have CMEs than those without. Falconer et al. (2002) showed that CME productivity of an AR depends on the global non-potentiality of its magnetic field (the length of the main neutral line, the net electric current, and the flux-normalized measure of the field twist). Švestka (2001) discussed the importance of open magnetic field in ARs for CME production. This study compares two flare-productive ARs in an AR-complex, with very different CME productivity. Identifying the distinguishing characteristics of the two ARs may help understand the presence and absence conditions of CMEs.

\section{$2 \quad$ AR 10039 and AR 10044}

Figure 1 shows AR 10039 and AR 10044 on the day of the central meridian passage (CMP) as visible in (a) the Michelson Doppler Imager (MDI) white light image, (b) the MDI magnetogram, and (c) EUV image obtained by the

yashiro@ssedmail.gsfc.nasa.gov (S. Yashiro), gopals@ssedmail.gsfc.nasa.gov (N. Gopalswamy). 
Extreme ultraviolet Imaging Telescope (EIT) on board the Solar and Heliospheric Observatory (SOHO). AR 10039 appeared at the east limb on July 21, 2002 and rotated out of the front side on August 4. This AR was magnetically complex, consisting of a spot group with a $\beta \gamma \delta$ configuration from the start. It produced $3 \mathrm{X}$ - and $8 \mathrm{M}$-class flares during the disk passage. The median X-ray flare intensity and duration were M5.3 and 29 minutes, respectively. AR 10044, located just to the southwest of AR 10039, emerged on July 23, 2002. It gradually developed into a $\beta \gamma$ delta on July 26, 2002. The two ARs formed an AR-complex. Nine M-class flares occurred in AR 10044 from July 26 to July 29, 2002. The median flare intensity and duration were M1.4 and 12 minute, weaker and shorter than those of AR 10039.

We used CME data obtained by the Large Angle and Spectrometric Coronagraph (LASCO) on board SOHO. We examined flare-CME associations by viewing both flare and CME movies. Eruptive surface activities, e.g. filament eruptions and dimmings, were searched to confirm the associations. Even though the two ARs formed an AR-complex, it was not difficult to find the AR source of CMEs because the flares were distinct with known flare locations. We also verified the flare location from the inner coronal images obtained by SOHO/EIT. Once the flare location is known, we identified the associated CME using flare-CME temporal coincidence. All flares can be divided by the CME associations into two groups: flares with and without CMEs. But there were some events for which the eruptive signature was not clear, so we introduced a third group: flare with uncertain CME association. We also excluded four flares because their CME associations could not be examined either due to LASCO data gaps or they occurred in the aftermath of preceding CMEs. Details on this analysis is described in Yashiro et al. (2005). The CME as- 
sociation rate (CME-R) of each AR was calculated by dividing the number of flares associated with CMEs by the total number of observed flares. The uncertain events were decided to have $50 \%$ possibility of CME association and used as the error bar. We found that AR 10039 was CME-rich with $72 \%$ ( $\pm 6 \%)$ flares having associated CMEs, while AR 10044 was CME-poor with a CME-R of only $14 \%( \pm 14 \%)$.

Figure 2a shows a CME originating from AR 10039 in association with an X3.3 class flare on July 20, 2002. Though the AR was located near the east limb, the CME was a halo with high speed $(1941 \mathrm{~km} / \mathrm{s})$. By contrast, the jet-like CME in Figure 2b on July 28, 2002 was associated with an M 2.3 flare from AR 10044. Another CME originating from AR 10044 was also thin and faint. We classified these CMEs as uncertain events, because they were too faint. The characteristics of CMEs originating from the two ARs seemed to be very different. We calculated the median velocity and the angular width of CMEs from the two ARs using the SOHO/LASCO CME Catalog ${ }^{1}$. We found that CMEs from the CME-rich AR were faster (median speed $=1195 \mathrm{~km} / \mathrm{s}$ ) and wider (median width $=246 \mathrm{deg}$ ) compared to those from the CME-poor AR $($ median speed $=282 \mathrm{~km} / \mathrm{s}$ and median width $=12 \mathrm{deg})$.

Properties of the two ARs are summarized in Table 1. First and second rows are the CMP time and the maximum sunspot area expressed in millionths of solar hemisphere (msh), respectively. Flare properties (total flare number, median X-ray intensity, median flare duration, and median flare waiting time) are listed from 3rd to 6th rows. The last three rows are CME properties (CME-R, median CME width, and median CME speed).

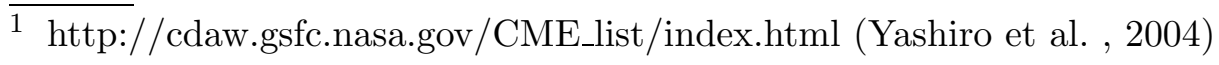




\section{Temporal Difference}

Figure 3 shows the GOES X-ray light curve during the disk passages. Flares in AR 10039 and AR 10044 are marked by letters "R" and "P", respectively. The flare categories are designated using filled circles (flares with CMEs), crosses (without CMEs), open circles (uncertain events), and triangles (events without good data coverage). Figure 4 shows the difference between the two ARs in terms of the waiting-time, which is the interval between peak times of successive flares (above M1 level). Note that the four excluded flares in Section 2 were included in this analysis. The flare waiting-time of AR 10039 (39.2 h) was much longer than that of AR $10044(5.1 \mathrm{~h})$. The flares from AR 10039 occurred about once a day all through the disk passage, while the flares from AR 10044 occurred in quick succession in a cluster lasting for only 2-3 days. This flaring spurt corresponded to the period of $\beta \gamma \delta$ configuration in AR 10044.

\section{Spatial Difference}

We examined the flare locations in the ARs using the EIT data during the passage from East 60 to West 60 degree. Limb flares (the longitude $>60$ degree) were eliminated because the magnetic configuration could not be determined accurately. Figure 5 shows the white light images obtained by the MDI from July 24 to July 31, 2002. We see that the flares in locations of AR 10039 occurred around its preceding or following spots, whereas those of AR 10044 were localized between the preceding and the following spots. No flare in AR 10039 occurred between the preceding and the following spots. The 
flare location seems to decide whether a flare is eruptive or confined at least for these regions.

We found that the spot structure of AR 10044 was much different at the beginning and end. New spots indicated by an arrow in Figure 5b emerged the same location where the flares occur. We recognized new spots as emerging flux, because the appearance of a small magnetic pair in this location was observed with the MDI magnetogram data. On the other hand, AR 10039 did not change dramatically. Thus the pre-eruption evolution was different for flares in the two ARs. Various types of pre-eruption evolution can occur on the Sun (Gopalswamy et al. , 2006). In AR 10044 it could be the flux emergence. In AR 10039 it might be a different mechanism.

\section{Summary}

We compared two flare-productive ARs with very different CME characteristics. AR 10039 had higher CME-R (72\%), while AR 10044 had lower CME$\mathrm{R}(14 \%)$. We found that flares in the CME-rich AR occurred intermittently (waiting time $\sim 39$ hours), while ones in the CME-poor AR occurred successively (waiting time $\sim 5$ hours). All flares originating from CME-poor AR were driven by the emerging flux between the preceding and the following spots. The place is favorable for flares to be confined by overlying magnetic fields. The longer pre-eruption evolution in AR 10039 seems to be consistent with more energy stored and released in the form of large CMEs. 


\section{References}

Canfield, R. H., Hudson, H. S., \& McKenzie, D. E. Sigmoidal morphology and eruptive solar activity. J. Geophys. Res. Letter, 26, 627-630, 1999.

Falconer, D. A., Moore, R. L., \& Gary, G. A. Correlation of the coronal mass ejection productivity of solar active regions with measures of their global nonpotentiality from vector magnetograms: baseline results. Astrophys. J., 569, 1016-1025, 2002.

Gopalswamy, N., Mikić, Z., Maia, D. et al. The pre-cme sun. Space Science Rev., 123, 303-339, 2006.

Švestka Z. Varieties of coronal mass ejections and their relation to flares. Space Sci. Rev. 95, 135-146, 2001.

Yashiro, S., Gopalswamy, N., Michalek, G. et al. A catalog of white light coronal mass ejections observed by SOHO spacecraft. J. Geophys. Res., 109, A07105, doi:10.1029/2003JA010282, 2004.

Yashiro, S., Gopalswamy, N., Akiyama, S. et al. Visibility of coronal mass ejections as a function of flare location and intensity. J. Geophys. Res., 110, A12S05, doi:10.1029/2005JA011151, 2005. 
Table 1

Basic Properties of ARs 10039 and 10044.

\begin{tabular}{|c|c|c|c|}
\hline & & AR 10039 & AR 10044 \\
\hline \multicolumn{2}{|c|}{ CMP Time } & 02/07/28 15:08 & 02/07/08 08:28 \\
\hline \multicolumn{2}{|c|}{ Max Spot Area $\left[\mathrm{mhs}^{2}\right]$} & $940(\mathrm{~S} 15 \mathrm{E} 59)$ & $780(\mathrm{~S} 21 \mathrm{~W} 76)$ \\
\hline \multirow[t]{4}{*}{ Flare } & Number & $\mathrm{X}-3, \mathrm{M}-8$ & M- 9 \\
\hline & Med. X-ray & M5.3 & M1.4 \\
\hline & Med. Duration [min] & 29 & 12 \\
\hline & Med. Waiting Time $[\mathrm{h}]$ & 39.2 & 5.1 \\
\hline \multirow[t]{3}{*}{$\mathrm{CME}$} & CME-R ${ }^{3}[\%]$ & $72( \pm 6)$ & $14( \pm 14)$ \\
\hline & Med. Width [deg] & 246 & 12 \\
\hline & Med. Velocity $[\mathrm{km} / \mathrm{s}]$ & 1195 & 282 \\
\hline
\end{tabular}

2) millionths of solar hemisphere is expressed in mhs. 3) Error bar information is calculated from the uncertain events. 


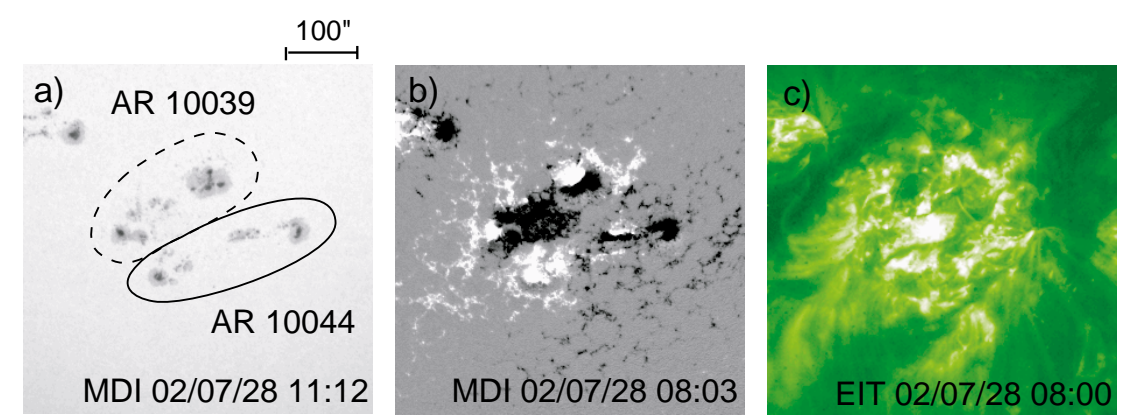

Fig. 1. An overview of AR 10039 (dashed line) and AR 10044 (solid line) on July 28, 2002, in by a) MDI white light image, b) MDI magnetogram, and c) EIT 195 Å image.
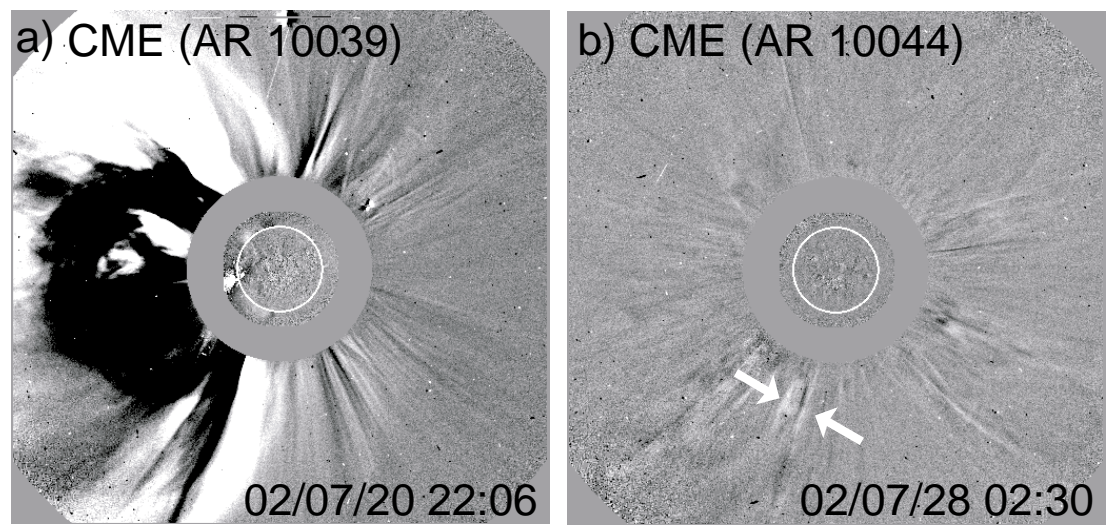

Fig. 2. a) The July 20, 2002 halo CME from AR 10039 detected by SOHO/LASCO. b) The July 28, 2002 CME from AR 10044, a faint jet-like structure shown between the two arrows. 


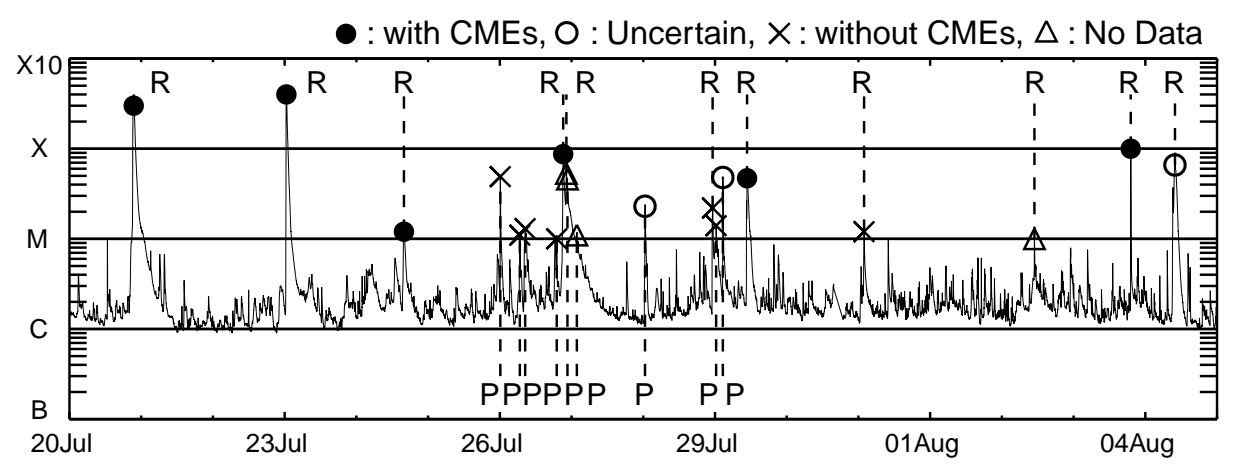

Fig. 3. GOES X-ray light curve during the disk passages of the two ARs. Strong flares ( $\geq$ M1) in AR 10039 and AR 10044 are marked R and P, respectively. AR 10039 is CME-rich (CME-R = 72\%), while AR 10044 is CME-poor (CME-P = $14 \%)$.

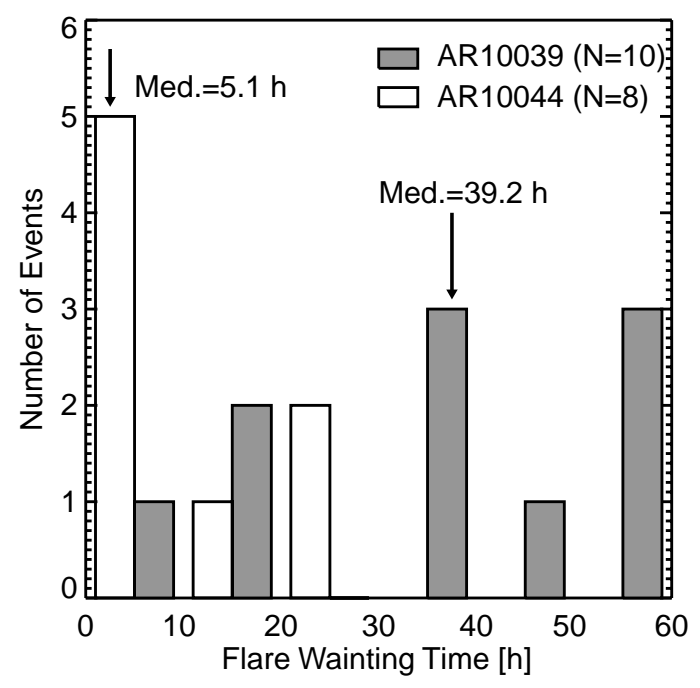

Fig. 4. Histogram of the flare waiting-times from AR 10039 (gray) and AR 10044 (white). Median values are indicated by the arrows. 
- : with CMEs, $O$ : Uncertain, $X$ : without CMEs, $\triangle$ : No Data

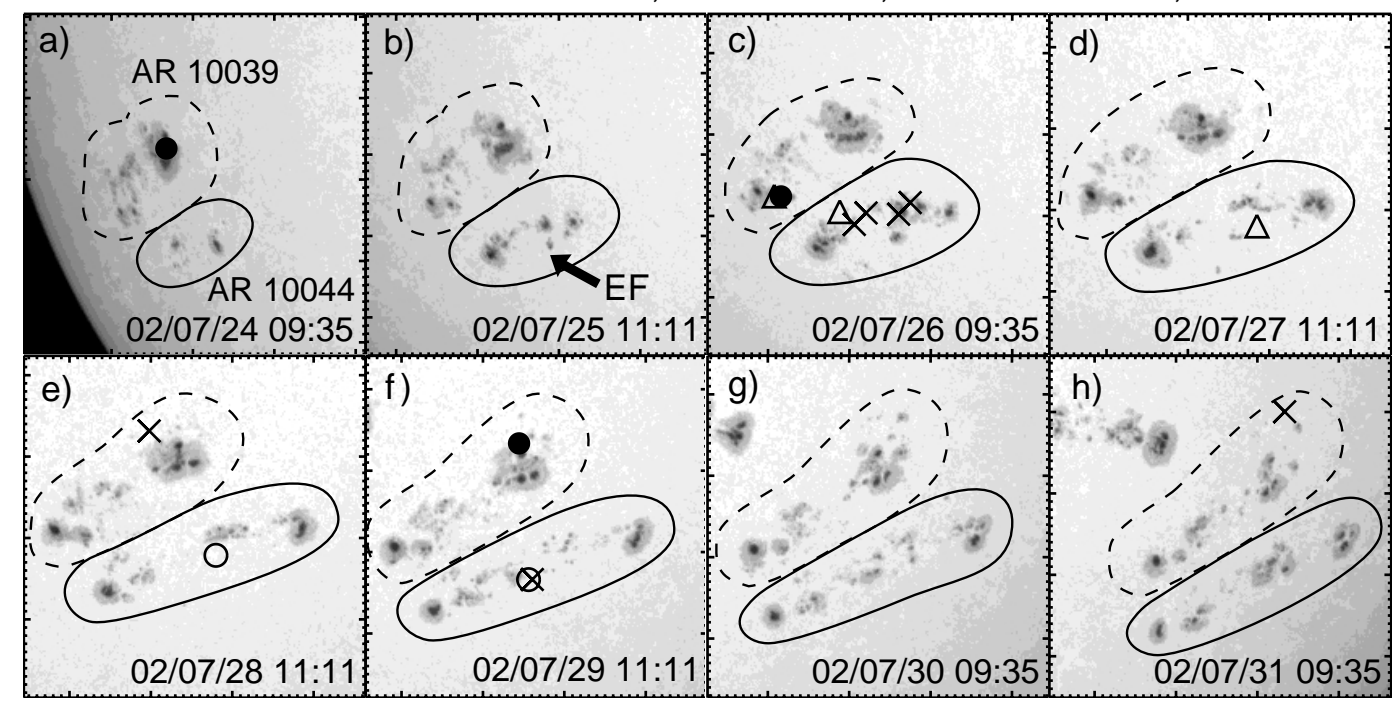

Fig. 5. White light images of AR 10039 (dashed line) and AR 10044 (solid line) obtained by SOHO/MDI from July 24 to July 31, 2002. Flare locations are marked by four symbols, which indicate the category of CME association. Newly emerging spots (EF) between the preceding and the following spots of AR 10044 are indicated by an arrow. AR 10039: Two flare locations, one near preceding spot, the other near following spot. AR 10044: All flares occurred in the same location - half way between the preceding and the following spots, where new flux emerged. 